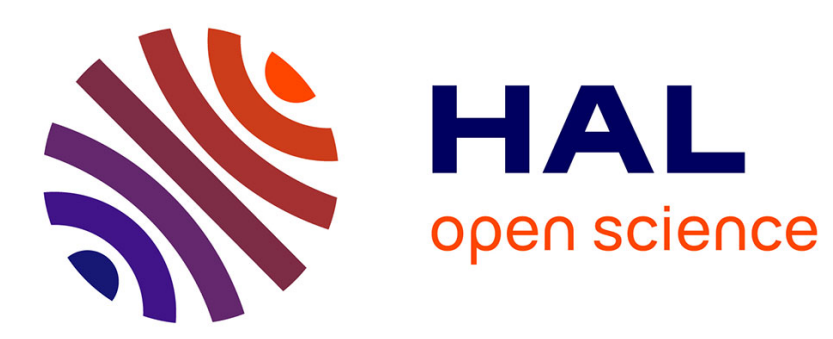

\title{
Some new aspects of the reflection of electromagnetic waves on a rough surface
}

\author{
Maurice A. Biot
}

\section{To cite this version:}

Maurice A. Biot. Some new aspects of the reflection of electromagnetic waves on a rough surface. Journal of Applied Physics, 1957, 28 (12), pp.1455-1463. 10.1063/1.1722676 . hal-01368684

\section{HAL Id: hal-01368684 https://hal.science/hal-01368684}

Submitted on 21 Sep 2016

HAL is a multi-disciplinary open access archive for the deposit and dissemination of scientific research documents, whether they are published or not. The documents may come from teaching and research institutions in France or abroad, or from public or private research centers.
L'archive ouverte pluridisciplinaire HAL, est destinée au dépôt et à la diffusion de documents scientifiques de niveau recherche, publiés ou non, émanant des établissements d'enseignement et de recherche français ou étrangers, des laboratoires publics ou privés. 


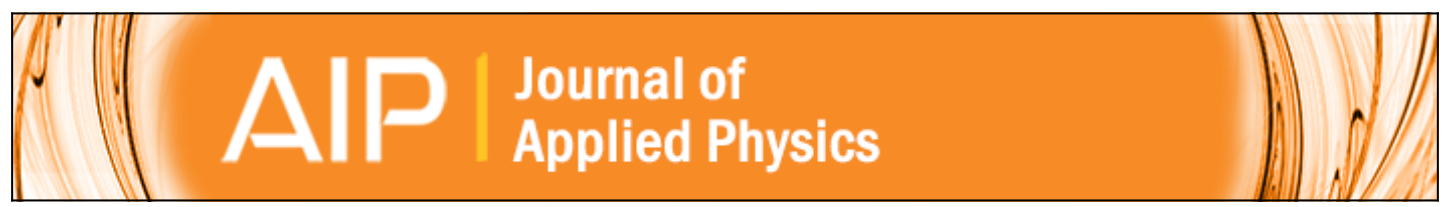

Some New Aspects of the Reflection of Electromagnetic Waves on a Rough Surface

M. A. Biot

Citation: Journal of Applied Physics 28, 1455 (1957); doi: 10.1063/1.1722676

View online: http://dx.doi.org/10.1063/1.1722676

View Table of Contents: http://scitation.aip.org/content/aip/journal/jap/28/12?ver=pdfcov

Published by the AIP Publishing

\section{Articles you may be interested in}

Some aspects of the adsorption of a Lennard-Jones gas on a rough surface

J. Chem. Phys. 110, 15 (1999); 10.1063/1.478078

Some aspects of the reflection and refraction of an electromagnetic wave at an absorbing surface

Am. J. Phys. 51, 245 (1983); 10.1119/1.13302

Reflection of Electromagnetic Waves from a Rough Surface

J. Appl. Phys. 36, 3609 (1965); 10.1063/1.1703050

On the Reflection of Electromagnetic Waves on a Rough Surface

J. Appl. Phys. 29, 998 (1958); 10.1063/1.1723349

On the Reflection of Acoustic Waves on a Rough Surface

J. Acoust. Soc. Am. 30, 479 (1958); 10.1121/1.1909658

\section{High Energy Nanosecond Lasers}
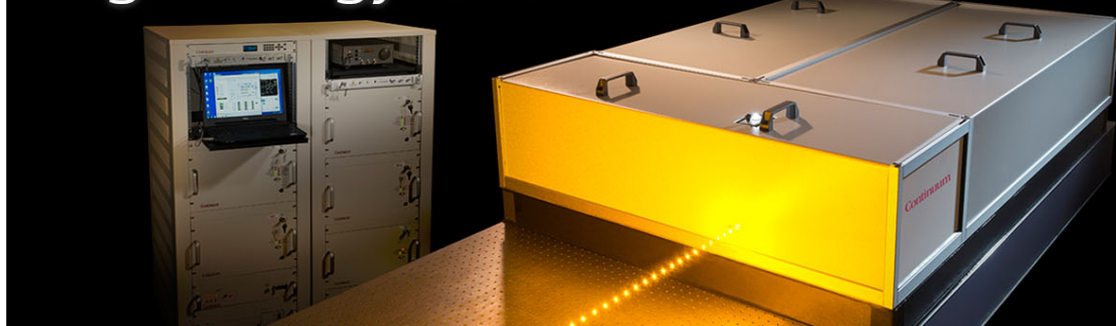


\title{
Some New Aspects of the Reflection of Electromagnetic Waves on a Rough Surface*
}

\author{
M. A. Brot \\ Cornell A eronautical Laboratory Inc., Buffalo, New York
}

(Received April 30, 1957)

\begin{abstract}
The reflection of a plane-electromagnetic wave from a rough perfect conductor is investigated. The roughness is represented by hemispherical bosses whose radii and mutual distances are small relative to the wavelength. This problem is solved taking into account the electromagnetic interaction of the bosses. Aside from throwing some light on the limitations of approximate methods, the present solution reveals some effects not known before. For grazing incidence and vertical polarization the interaction has a drastic influence in that it causes a complete phase reversal of the reflected wave, near a 45 degree incidence the influence of the roughness vanishes. These effects do not occur for horizontal polarization. An approximate treatment for the case of the imperfect conductor is indicated. The analogous case for the acoustic wave is also developed and shows similar behavior. The effect of the roughness is shown to be equivalent to a boundary condition for the wave equation. Extension of the method to a more general type of roughness, and to the case of statistical and asymmetric shapes and distributions, is briefly discussed.
\end{abstract}

\section{INTRODUCTION}

$T$ HE problem of reflection of an electromagnetic wave from a rough surface may be conveniently treated if we represent the roughness by hemispherical bosses distributed over the surface. An exact solution for the problem of a single hemispherical boss on a perfect conductor is immediately derived from that of scatter of plane waves by an isolated sphere. It is easily seen that the problem is solved by an image method in the plane of reflection. The problem is considerably simplified if we introduce the assumption that the radius of the sphere is small relative to the wavelength. In Sec. 2, it is pointed out that the wave scattered by the sphere is identical with that radiated by an electrical and a magnetic dipole located at its center. Small hemispherical bosses on a reflecting surface are then equivalent to a distribution of electric and magnetic dipoles. The problem of reflection of a plane wave form such a surface implies the solution of an integral equation, which expresses the fact that the radiation of a single boss depends on the integrated radiation of all the others. It is shown in Sec. 3 how an exact solution of the integral equation may be found when the distribution of the roughness is uniform, and when it is dense; i.e., when the distance between the bosses is small relative to the wavelength. Section 7 discusses the extension of the theory for the case where the mutual distance of the bosses is of the order of their radii. In such a case the phenomenon is not properly in the nature of a scatter, and there is no energy dispersion. The main effect may be described as caused by an induced electric and magnetic polarization of the surface roughness, which produces a phase shift of the reflected wave.

\footnotetext{
* The contents of this paper first appeared as part of Cornell Aeronautical Laboratory, Inc. report No. BE-745-T-129 dated March, 1955 (see reference 1). The work was performed under contract DA-30-115-ORD-47, with the Rochester Ordnance District Department of the Army.

+ Consultant
}

The case of vertical polarization of the incident wave, treated in Sec. 4, shows that for grazing incidence the effect is quite drastic, and that the phase shift reaches 180 degrees. At 45 degrees incidence a cancellation of the effect of the roughness takes place. Section 5 deals with the case of horizontal polarization where no such effect is found for grazing incidence.

The method of the present paper may be extended to find approximate solutions for the reflection on an imperfect conductor or dielectric. For instance, in the case of vertical polarization and grazing incidence, vertical electric dipoles are induced at the surface, the magnitudes of which are evaluated in reference 1 , (Appendix B). This approximate evaluation is carried out in Sec. 6, and leads to properties of the reflected waves quite similar to that of the perfect conductor, and the same grazing angle effect.

That the above features are not restricted to electromagnetic phenomena is shown by treating the analogous case of an acoustic wave falling upon a rough surface of small, densely distributed, hemispherical bosses. It is shown that this is identical with the case of electromagnetic waves when vertical electric dipoles only are induced in the roughness. The same complete phase reversal is also exhibited by an acoustic wave at grazing incidence.

These results indicate that this grazing incidence effect, which is a direct consequence of the interaction between the roughness elements, represents some kind of resonance between the incident wave and the propagating interactions. The fact that it occurs at grazing incidence is understood if we consider that for such incidence the phase velocity of the incident wave along the reflecting surface is nearly equal to the

${ }^{1}$ M. A. Biot, Reflection of an electromagnetic wave from a surface with small roughness, Cornell Aeronautical Laboratory, Inc. Report No. BE-745-T-129, March, 1955, Appendix A, "General discussion of electrodynamics when dimensions are small relative to the wavelength," Appendix B, "Scatter of a plane wave by a spherical imperfect conductor." 
characteristic velocity of propagation of the medium. The signals generated by the roughness will tend to accumulate in the direction of propagation.

The methods introduced here are easily extended to other cases than plane waves. As further applications, the reflection of electromagnetic dipole radiation from a rough surface has been expressed in closed form, ${ }^{2}$ as well as the reflection on a rough surface from an acoustic point-source. $^{3}$

The representation of roughness by hemispherical bosses on a perfect conductor has been used by Twersky ${ }^{4}$ who derived the energy scattered by one hemisphere, and integrated the effect of a sparse distribution of scattering centers neglecting the interaction between bosses. A different approach was made by Rice $^{5}$ who considers a slightly rough surface of random distribution and approximates the solutions up to the second order in the magnitude of the roughness. The present exact solution, although restricted to the case of a dense and uniform distribution of roughness, throws some light on the limitations of the approximate methods, particularly at grazing incidence.

As indicated in Sec. 7, the present theory is easily extended to more general types of roughness than hemispherical bosses by taking into account the local geometry. This local geometry appears in the form of coefficients which depend on the shape and mutual distances of the protuberances, and on the statistical correlation of these parameters. Higher order induced multipoles which appear in a more general theory may also be introduced by generalizing the boundary condition to include higher derivatives. The effect of anisotropy due to either shape or statistical distribution and causing directional properties may also be introduced. It is pointed out that this should lead to interesting conclusions regarding the effect of ocean waves on radio transmission. The present theory also separates clearly the coherent and noncoherent scatter. It suggests the possibility of treating the latter as a perturbation superposed on the coherent field derived hereafter, and it should improve the physical interpretation of the mathematical treatment of the more general problem.

\section{FIELD SCATTERED BY A PERFECTLY CONDUCTING SPHERE}

Consider a plane electromagnetic wave propagating in the $x$ direction, the plane of polarization being $x z$ (Fig. 1). A sphere of radius $a$ located at the origin produces a scattered wave. We assume that the radius of the sphere is small relative to the wavelength, and

${ }^{2}$ M. A. Biot, "A closed-form solution for the reflection of electromagnetic dipole radiation on a rough surface," Cornell Aeronautical Laboratory, Inc., Report (March, 1955) (to be published).

${ }^{3}$ M. A. Biot, J. Acoust. Soc. Am. 29, 1193-1200 (1957).

${ }^{4}$ V. Twersky, J. Appl. Phys. 22, 825-835 (1951).

5 S. O. Rice, "Reflection of electromagnetic waves from slightly rough surfaces" in Symposium on the Theory of Electromagnetic Waves (Interscience Publishers, Inc., New York, 1951). that its conductivity is infinite. Because of the assumption of a small radius, the incident field may be considered uniform in the vicinity of the sphere. The problem is then to add a perturbation field such that at the surface of the sphere to total magnetic field be tangent to the surface, and the total electric field be normal. We will show that this condition is satisfied if we locate at the center of the sphere a radiating electric dipole parallel to the incident electric field, and a radiating magnetic dipole parallel to the incident magnetic field. The electric Hertz vector $\pi$, caused by the electric dipole, is oriented along $z$,

$$
\pi=\left(0,0, \pi_{z}\right) \text {. }
$$

The magnetic Hert $z$ vector $\boldsymbol{\pi}^{*}$, caused by the magnetic dipole is oriented along $y$,

$$
\boldsymbol{\pi}^{*}=\left(0, \boldsymbol{\pi}^{*}, 0\right) \text {. }
$$

As a function of the distance $r$ from the origin and the dipole moments $M$ and $M^{*}$ we have

$$
\begin{aligned}
\pi_{z} & =M\left(e^{-i k r} / r\right), \\
\pi_{y}{ }^{*} & =M^{*}\left(e^{-i k r} / r\right) .
\end{aligned}
$$

The field radiated by these dipoles is given by ${ }^{6}$

$$
\begin{aligned}
\mathbf{E}^{\prime} & =\operatorname{grad} \operatorname{div} \boldsymbol{\pi}+k^{2} \boldsymbol{\pi}-i k \operatorname{curl} \boldsymbol{\pi}^{*}, \\
\mathbf{H}^{\prime} & =\operatorname{grad} \operatorname{div} \boldsymbol{\pi}^{*}+k^{2} \boldsymbol{\pi}^{*}+i k \operatorname{curl} \boldsymbol{\pi} .
\end{aligned}
$$

The following quantities are introduced: $k=\omega / c=2 \pi / \lambda$ $=$ wave number $; \omega=$ circular frequency; $\lambda=$ wavelength; $c=$ velocity of light. The dielectric constant of the surroundings is assumed to be that of vacuum. Since the sphere radius is assumed to be small, we are interested only in the near field, i.e., the field in the vicinity of the origin. It is readily verified that the near field is the same as for a static dipole (case $k=0$ ). This is discussed more extensively in reference 1 (Appendix A). Hence this field is

$$
\begin{aligned}
\mathbf{E}^{\prime} & =\operatorname{grad} \operatorname{div} \boldsymbol{\pi}, \\
\mathbf{H}^{\prime} & =\operatorname{grad} \operatorname{div} \boldsymbol{\pi}^{*},
\end{aligned}
$$

with

$$
\begin{gathered}
\pi_{z}=M / r, \\
\pi_{y}^{*}=M^{*} / r .
\end{gathered}
$$

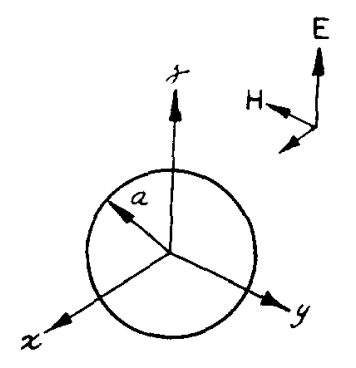

FiG. 1. Plane-wave incident upon a sphere.

${ }^{6} \mathrm{~J}$. A. Stratton, Electromagnetic Theory (McGraw-Hill Book Company, Inc., New York, 1941), p. 31 (converting to Gaussian units). 
We have

$$
\begin{aligned}
\operatorname{div} \pi & =-\left(M z / r^{3}\right)=-\left(M / r^{2}\right) \cos \theta, \\
\operatorname{div} \pi^{*} & =-\left(M y^{*} / r^{3}\right)=-\left(M^{*} / r^{2}\right) \cos \theta^{*},
\end{aligned}
$$

where $\theta$ and $\theta^{*}$ are azimuthal angles of $\mathbf{r}$ with the $z$ and $y$ axes, respectively (Fig. 2). From (2.5) the tangential component of the electric dipole field at $r=a$ is

$$
E_{\theta}{ }^{\prime}=\left(M / a^{3}\right) \sin \theta
$$

and the normal component of the magnetic field

$$
H_{r}^{\prime}=\left(2 M^{*} / a^{3}\right) \cos \theta^{*} .
$$

From these expressions we see that it is possible to cancel the tangential component of the electric field and the normal component of the magnetic field by putting

$$
\begin{gathered}
M=E a^{3}, \\
M^{*}=\frac{1}{2} H a^{3} .
\end{gathered}
$$

It can be shown that the field scattered by these two dipoles leads to the expression obtained by Twersky by a more elaborate derivation. ${ }^{4}$

A similar reasoning will yield the scatter by a dielectric or imperfectly conducting sphere. The imperfect conductibility cancels the magnetic dipole and we are left with only the electric dipole of the wellknown Rayleigh solution. For the Rayleigh solution to apply, the so-called skin depth (which is a measure of the depth of penetration of the wave) must be large relative to the sphere radius. ${ }^{7}$ If this condition is not fulfilled, a magnetic dipole must be added of a magnitude intermediate between zero and the full value (2.10). This is discussed more extensively in reference 1 (Appendix B).

\section{REFLECTING PROPERTIES OF DENSELY DISTRIBUTED HEMISPHERICAL BOSSES ON A PERFECTLY CONDUCTING SURFACE}

It is easily verified that the field scattered by a hemispherical boss on a plane perfect conductor is the same as that scattered by a complete sphere under the incident wave and its image with respect to the plane. Consider the total imposed field, incident plus reflected, near the plane conductor in the absence of the boss. This

FIG. 2. Components of the near-field.

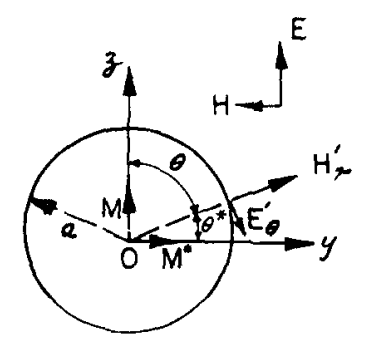

${ }^{7}$ M. A. Biot, Doctoral dissertation, Louvain University, 1931; Ann. soc. sci. Bruxelles Ser. B, 51, 94-127 (1931).
FIG. 3. Hemispherical boss on a reflecting plane.

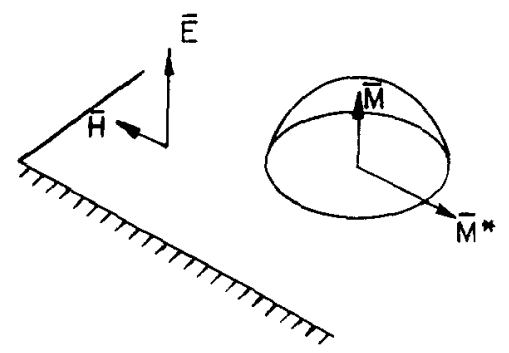

is an approximately uniform field with a normal electric component $\mathbf{E}$ and a tangential magnetic component $\mathbf{H}$ (Fig. 3). The boundary condition at the surface of the hemispherical boss will be satisfied by placing at the center of the sphere a tangential magnetic dipole of moment $\mathbf{M}^{*}$ parallel and opposite to $\mathbf{H}$, and a normal electric dipole of moment $\mathbf{M}$ parallel and in the same direction as $\mathbf{E}$. The magnitudes of these moments are given by (2.10) with the addition of the proper sign.

$$
\begin{aligned}
\mathbf{M} & =\mathbf{E} a^{3}, \\
\mathbf{M}^{*} & =-\left(\mathbf{H} a^{3} / 2\right) .
\end{aligned}
$$

We assume now that there is a dense distribution of hemispherical bosses such that the number of scattering centers per unit area is $N$. The electric and magnetic Hertz vectors are given respectively by the integrals over the surface $S$ of the reflecting plane

$$
\begin{aligned}
\boldsymbol{\pi} & =N \iint \mathbf{M}\left(e^{-i k r} / \boldsymbol{r}\right) d S, \\
\boldsymbol{\pi}^{*} & =N \iint \mathbf{M}^{*}\left(e^{-i k r} / \boldsymbol{r}\right) d S .
\end{aligned}
$$

However, the dipole moments are not known since they depend on the values of both the imposed field and the field induced by the dipoles themselves. Hence,

$$
\begin{aligned}
\mathbf{M} & =\left(\mathbf{E}+\mathbf{E}^{\prime}\right) a^{3}, \\
\mathbf{M}^{*} & =-\frac{1}{2}\left(\mathbf{H}+\mathbf{H}^{\prime}\right) a^{3},
\end{aligned}
$$

when $\mathbf{E}^{\prime}$ and $\mathbf{H}^{\prime}$ represent the field radiated by the dipoles themselves. Equations (3.2) and (3.3) together amount to a pair of integral equations for the unknown distribution of the dipole density. The problem of solving these integral equations may be transformed into one of solving the wave equation with a boundary condition on the reflecting surface, which we shall now derive. The vectors $\pi$ and $\pi^{*}$ both satisfy the wave equation,

$$
\square \pi=\square \pi^{*}=0 .
$$

If we now consider a general integral of the type (3.2) (which satisfies the wave equation)

$$
\varphi=\iint \frac{e^{-i k r}}{r} d S,
$$

and is taken over the plane $x, y$, we may verify that the 


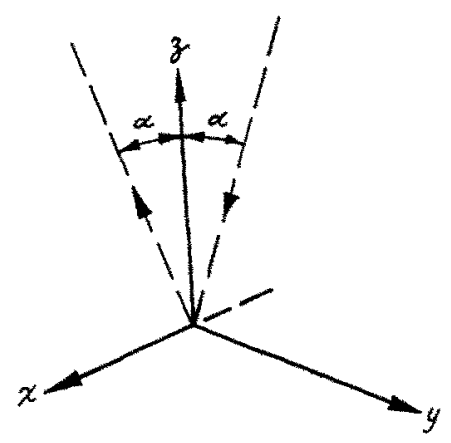

normal derivative $\partial \varphi / \partial z$ very close to the plane is the same as if the sources were potential sources $1 / r$ instead of radiation sources $e^{-i k r} / r$. Hence, by the potential theory at $z=0$

$$
(\partial \varphi / \partial z)=2 \pi \rho
$$

Applying this property to the integrals (3.2) we find a boundary condition at the reflecting surface

$$
\begin{gathered}
\partial \pi / \partial z=2 \pi N \mathbf{M}, \\
\partial \pi^{*} / \partial z=2 \pi N \mathbf{M}^{*} .
\end{gathered}
$$

Introducing (3.3) into (3.7) and the parameter

$$
\sigma=2 \pi N a^{3},
$$

the boundary condition is written

$$
\begin{gathered}
(\partial \pi / \partial z)=\sigma\left(\mathbf{E}+\mathbf{E}^{\prime}\right), \\
\left(\partial \pi^{*} / \partial z\right)=-\frac{1}{2} \sigma\left(\mathbf{H}+\mathbf{H}^{\prime}\right) .
\end{gathered}
$$

The paremeter $\sigma$ has the dimension of a length. We may write

$$
\sigma=3 \tau,
$$

where $T$ represents the volume of the hemispherical bosses per unit area. The radii of all the spheres do not have to be the same since we may average out their volume provided the density may still be considered uniform in comparison with the magnitude of the wavelength.

The boundary condition (3.9) may also be considered as representing an electric and magnetic polarization of the surface.

\section{REFLECTION OF A VERTICALLY POLARIZED WAVE}

We consider a plane wave reflected on the $x, y$ plane, its plane of polarization being $x, z$ with an angle of incidence $\alpha$ (Fig. 4). The representation of resultant field resulting from reflection on a perfect conductor may now be derived from the following Hertz vectors:

$$
\begin{aligned}
\pi & =\left(0,0, \pi_{z}\right), \\
\boldsymbol{x}^{*} & =\left(0, \boldsymbol{\pi}_{\mathbf{y}}^{*}, 0\right),
\end{aligned}
$$

The components $\pi_{z}$ and $\pi_{z}^{*}$ are functions of $x$ and $z$ only and satisfy the wave equations

$$
\begin{array}{r}
\frac{\partial^{2} \pi_{z}}{\partial x^{2}}+\frac{\partial^{2} \pi_{z}}{\partial z^{2}}+k^{3} \pi_{z}=0, \\
\frac{\partial^{2} \pi_{y}{ }^{*}}{\partial x^{2}}+\frac{\partial^{2} \pi_{y}{ }^{*}}{\partial z^{2}}+k^{2} \pi_{y}{ }^{*}=0 .
\end{array}
$$

Applying (2.4) and taking into account (4.2) we find for the field components

$$
\begin{array}{lll}
E_{x}=(\partial V / \partial z) & E_{y}=0 & E_{z}=-(\partial V / \partial x), \\
H_{x}=0 & H_{y}=-i k V & I_{z}=0,
\end{array}
$$

with the introduction of a scalar

$$
V=\left(\partial \pi_{z} / \partial x\right)+i k \pi_{y}^{*}
$$

satisfying the wave equation $(4,2)$.

The field resulting from the incident and reflected wave on a smoolh perfectly conducting surface is represented by

with

$$
V=D\left(e^{i m s}+e^{-i m_{z}}\right) e^{-i l x},
$$

$$
k^{2}=m^{2}+l^{2} \quad m>0,0<l<k .
$$

If the roughness is represented by a dense and uniform distribution of hemispherical bossess, we must add to the wave system (4.5) the plane wave resulting from the radiation of these bosses. The distribution of electric and magnetic dipoles equivalent to the roughness, generates a wave given by its electric and magnetic Hert $z$ vectors $\pi^{t}, \pi^{* r}$ parallel with the vectors (4.1). Hence, we may represent it by the scalar $V^{\prime}$

$$
V^{\prime}=\left(\partial \pi_{z}^{\prime} / \partial x\right)+i \pi_{y}^{* \prime}
$$

The boundary condition (3.9) is now written with a prime notation to indicate that the Hertz vectors are those of the wave generated by the roughness alone

$$
\begin{gathered}
\left(\partial \pi_{z}{ }^{\prime} / \partial z\right)=\sigma\left(E_{z}+E_{z}{ }^{\prime}\right), \\
\left(\partial \pi_{z}{ }^{*} / \partial z\right)=-\frac{1}{2} \sigma\left(H_{y}+H_{y}{ }^{\prime}\right) .
\end{gathered}
$$

Eliminating $\pi_{z}{ }^{\prime}$ and $\pi_{z}{ }^{* \prime}$ from (4.6) and (4.7) we find

$$
\left(\partial V^{\prime} / \partial z\right)=\sigma(\partial / \partial x)\left(E_{z}+E_{z}{ }^{\prime}\right)-(i k \sigma / 2)\left(H_{y}+H_{y}{ }^{\prime}\right),
$$

or

$$
\frac{\partial V^{\prime}}{\partial z}=-\sigma\left(\frac{\partial^{2}}{\partial x^{2}}+\frac{1}{2}\right)\left(V+V^{\prime}\right)
$$

Let us take $V^{\prime}$ to represent a plane outgoing wave

$$
V^{\prime}=\mathrm{Ce}^{-i m z} e^{-i l x}
$$

with a complex amplitude $C$ to be determined. Substituting $V$ and $V^{\prime}$ in the boundary condition (4.9) for $z=0$ and solving for $C$ we find

$$
C=2 D \frac{\sigma\left(m^{2}-l^{2}\right)}{2 i m-\sigma\left(m^{2}-l^{2}\right)} .
$$


The total amplitude of the reflected wave including the effect of the roughness is

$$
C+D=D \frac{2 m+i \sigma\left(l^{2}-m^{2}\right)}{2 m-i \sigma\left(l^{2}-m^{2}\right)} .
$$

Introducing the angle of incidence $\alpha$ we write

$$
\begin{gathered}
l=k \sin \alpha, \\
m=k \cos \alpha .
\end{gathered}
$$

Hence,

$$
C+D=D \frac{2 \cos \alpha-i \sigma k \cos 2 \alpha}{2 \cos \alpha+i \sigma k \cos 2 \alpha} .
$$

This may also be written

with

$$
C+D=D e^{2 i \varphi},
$$

$$
\tan \varphi=-\frac{1}{2} \sigma k(\cos 2 \alpha / \cos \alpha)
$$

We see that the amplitude of the total reflected wave is the same as for the incident wave which is as it should be for conservation of energy. There is, however, a phase change $2 \varphi$ given by (4.15).

The controlling parameter is $\frac{1}{2} \sigma k$ which is in the nature of a ratio of the length $\sigma$ to the wavelength. This ratio will usually be small; hence, also the effect of the roughness. However, in the case of grazing incidence where $\alpha$ is close to $\pi / 2$ the effect is large and the phase angle of the reflected wave is changed by 180 degrees. It is also interesting to note that for an incidence of 45 degrees we find $\varphi=0$, and for that case the roughness has no effect because of mutual cancellation of the induced electric and magnetic dipole radiations.

The large effect of roughness at grazing incidence can be understood if we consider that it arises from some sort of resonance effect between the roughness interactions and the incident wave. At grazing incidence the phase velocity of the incident wave along the surface is near the velocity of propagation of the interaction signals between roughness elements. The effect is seen to disappear if the interaction is neglected as discussed below.

Going back to expression (4.14) we expand it to the first order in $\sigma k \cos 2 \alpha / \cos \alpha$

$$
C+D=D\left[1-i \sigma k \frac{\cos 2 \alpha}{\cos \alpha}\right] .
$$

This solution would have been obtained directly by neglecting the interaction between the hemispherical bosses. The present exact results show that this firstorder solution (4.16) breaks down at grazing incidence no matter how small the parameter $\sigma k$.

From the approximate formula (4.16) we write

$$
C+D=R e^{i \psi}
$$

FIG. 5. Assumed distribution of bosses.

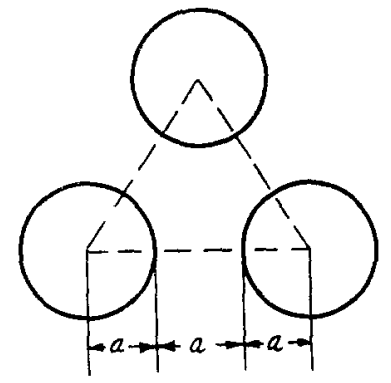

with

and

$$
R=D\left[1+\left(\sigma k \frac{\cos 2 \alpha}{\cos \alpha}\right)^{2}\right]^{\frac{1}{2}},
$$

$$
\tan \psi=-\sigma k(\cos 2 \alpha / \cos \alpha) .
$$

These expressions are correct to the first order in $\sigma k \cos 2 \alpha / \cos \alpha$. If we compare the approximate formula (4.19) with the exact one (4.15), we see that the error results in putting

$$
\tan 2 \varphi \cong 2 \tan \varphi .
$$

Hence, the first order theory gives a valid expression in so far as the phase angle $2 \varphi$ may be put equal to its tangent.

It is of interest to examine the magnitude of the basic quantity $\sigma k$. If $\lambda$ denotes the wavelength of the incident wave, we find

$$
\sigma k=6 \pi(\tau / \lambda) .
$$

The factor $6 \pi$ makes this quantity appreciable even when $\tau / \lambda$ is of the order of $10^{-2}$. The value of $\tau / \lambda$ depends not only on the size of the bosses but on the density of their distribution. We have considered the case when centers of the hemispheres are located at the vertices of a network of equilateral triangles of sides $3 a$ (Fig. 5). In this case the value of $\tau$ is related to the radius $a$ by

Hence,

$$
\tau=\frac{4 \pi}{27 \sqrt{3}} a .
$$

$$
\sigma k=\frac{8 \pi^{2} a}{9 \sqrt{3} \lambda} \underset{\lambda}{\simeq} \underset{\lambda}{\lambda} .
$$

With this value of $\sigma k$ the phase angle $2 \varphi$ of the reflected wave has been calculated by the exact formula (4.15) for the following ratios of the radius to the wavelength

$$
a / \lambda=1 / 10,1 / 25,1 / 50 \text {. }
$$

Results are plotted in Fig. 6 as a function of the angle of incidence $\alpha$. It can be seen that the breakdown of the first-order theory and the grazing incidence effect may occur at angles of incidence as low as 60 degrees, depending on the value of $a / \lambda$.

It is of interest to examine the possibility that the present type of roughness might render possible the existence of a surface wave for a perfect conductor. 


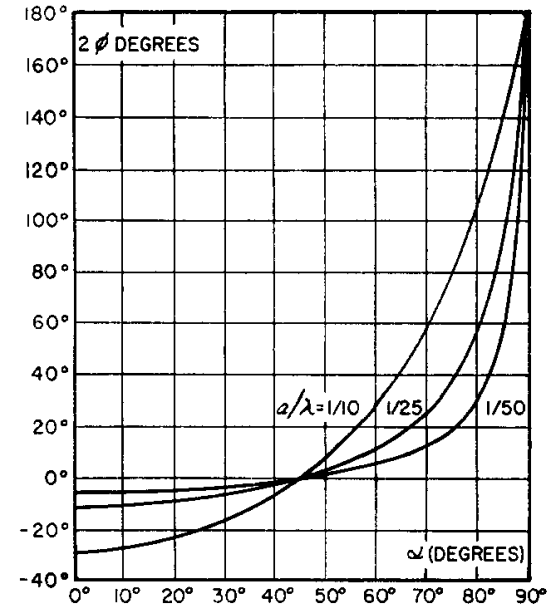

FIG. 6. Phase angle $2 \varphi$ of the reflected wave as a function of the angle of incidence.

This would mean the existence of a solution of the type

$$
V^{\prime}=C e^{-B z} e^{-i l x},
$$

with $\beta$ real and positive, and that such a solution would exist in case no incident field is present, i.e., $V=0$. The boundary condition (4.9) becomes

$$
\frac{\partial V^{\prime}}{\partial z}=-\sigma\left(\frac{\partial^{2}}{\partial x^{2}}+\frac{1}{2} k^{2}\right) V^{\prime} .
$$

Since (4.25) satisfies the wave equation we have

$$
k^{2}+\beta^{2}=l^{2} .
$$

Substituting (4.25) and (4.27) in (4.26) we find

$$
\beta^{2}+(2 \beta / \sigma)+l^{2}=0,
$$

which does not have a positive real root for $\beta$. Hence, under our assumption on the nature of the roughness, an unattenuated surface wave does not exist.

It is interesting to note that for normal incidence the phase $2 \varphi$ of the reflected wave is negative and contrary to expectation the influence of the roughness is equivalent to lowering the effective reflecting surface relative to the original plane surface without bosses. This is because at normal incidence only the magnetic field is operative at the surface in creating a dipole moment. A raising of the effective plane of reflection would occur if we introduced the higher order induced electric multipole as suggested in Sec. 7.

\section{REFLECTION OF A HORIZONTALLY POLARIZED WAVE}

We shall now consider a plane horizontally polarized wave incident upon a perfect conductor with hemispherical bosses. If we consider first a smooth surface, the electric field vanishes in the vicinity of the surface and we have only a magnetic field parallel with the surface. This magnetic field is directed along the $x$ axis and induces a horizontal magnetic dipole in this direction at the center of the hemispherical boss. No electric dipole is induced. The wave, incident and reflected on a smooth surface, is represented by a magnetic Hertz vector directed along the $x$ axis and of magnitude

$$
\pi_{x}^{*}=D\left(e^{i m z}+e^{-i m z}\right) e^{-i l x} \text {. }
$$

The magnetic Hertz vector resulting from the radiation of the hemispherical bosses is

$$
\pi_{x}{ }^{*}=C e^{-i m a} e^{-i l x}
$$

From the boundary condition (3.9) we write at $z=0$

$$
\frac{\partial \pi_{x}^{*}}{\partial z}=-\frac{1}{2} \sigma\left(H_{x}+H_{x}{ }^{\prime}\right),
$$

where the magnetic field components are

$$
\begin{aligned}
H_{x} & =\frac{\partial^{2} \pi_{x}{ }^{*}}{\partial x^{2}}+k^{2} \pi_{x}{ }^{*}=-\frac{\partial^{2} \pi_{x}}{\partial z^{2}} \\
H_{x}{ }^{\prime} & =-\partial^{2} \pi^{*} / \partial z^{2} .
\end{aligned}
$$

Hence, the boundary condition reduces to

$$
\frac{\partial \pi_{x}{ }^{*}}{\partial z}=\frac{1}{2} \sigma \frac{\partial^{2}}{\partial z^{2}}\left(\pi_{x}{ }^{*}+\pi_{x}{ }^{*}\right) .
$$

Substituting (5.1) and (5.2) yields the amplitude of the wave radiated by the roughness

$$
C=-2 D i \sigma m /(2+i \sigma m)
$$

and the total reflected wave

$$
C+D=D(2-i \sigma m) /(2+i \sigma m) .
$$

Again we may introduce a phase angle and write

with

$$
C+D=D e^{2 i \varphi},
$$

$$
\tan \varphi=-(\sigma m / 2) .
$$

From (4.13) we may also write

$$
\tan \varphi=-\frac{1}{2} \sigma k \cos \alpha .
$$

In this case of horizontal polarization the effect of the roughness is generally small since $\varphi$ will be of the order of $\sigma k$. It is maximum for normal incidence and disappears at grazing incidence. It may be also verified that no surface wave exists in case of horizontal polarization.

Disappearance of the grazing incidence effect in this case is understandable since the exciting field $H_{x}$ tends to disappear for those incidences like $\cos ^{2} \alpha$.

Results of this section and the previous one lead to the conclusion that an incident wave with oblique polarization falling on a rough perfect conductor will produce a reflection which, in general, will be elliptically polarized. 


\section{APPROXIMATE TREATMENT FOR FINITE CONDUCTIVITY AND THE ANALOGOUS ACOUSTIC CASE}

It is possible to use the methods developed above to treat approximately the case of a nonperfect conductor. In order to illustrate this let us assume that we are dealing with a case of grazing incidence and vertical polarization, and that the wave system existing in the absence of roughness is such that the energy is completely reflected in first approximation. This wave system is then represented by the scalar (4.5), and the amplitude of the incident wave is represented by $D$. The difference of this case with that of the perfect conductor lies in the fact that the magnetic dipole induced by the field in the hemispherical boss will be reduced in magnitude and will completely vanish if the conductivity or frequency is low enough, so that the skin depth is much larger than the hemispherical boss. Then only a vertical electric dipole is induced in the roughness. The moment of this dipole for low conductivity or frequency is the same as that given by the first expression (3.1) (see reference 1, Appendix B).

$$
M=E_{z} a^{a} .
$$

In the case of a material of high dielectric constant $K$ the electric moment induced is

$$
M=[(K-1) /(K+2)] E_{z} a^{3} .
$$

Equations (4.7) and (4.9) for the boundary conditions are then replaced by

$$
\begin{aligned}
& \partial \pi_{z}^{\prime} / \partial z=\sigma\left(E_{z}+E_{z}^{\prime}\right), \\
& \partial V^{\prime} / \partial z=-\sigma\left(\partial^{2} / \partial x^{2}\right)\left(V+V^{\prime}\right),
\end{aligned}
$$

where

$$
\text { or } \begin{gathered}
\sigma=2 \pi N a^{3}, \\
\sigma=2 \pi N \frac{K-1}{K+2} a^{3},
\end{gathered}
$$

depending on whether we use expression (6.1) or (6.2). The wave radiated by the roughness is $V^{\prime}$ as in $\mathrm{Eq}$. (4.1), and its amplitude $C$ is found by substitution in the boundary condition (6.3). We find for the total reflected wave

$$
C+D=\frac{m+i \sigma l^{2}}{m-i \sigma l^{2}} D
$$

This may be written

with

$$
C+D=D e 2^{i \varphi},
$$

$$
\tan \varphi=\sigma k\left(\sin ^{2} \alpha / \cos \alpha\right) .
$$

We notice that in this case too there is a drastic influence of the roughness at grazing incidence where the phase angle of the reflected wave changes by 180 degrees. However, in contrast to the perfect conductor, the effect of the roughness does not disappear for an incidence of 45 degrees.

It is of interest to consider the corresponding acoustic case of a plane-acoustic wave falling upon a rough surface with a dense and uniform distribution of small hemispherical bosses of radius $a$. It turns out that the treatment of this problem is exactly the same as the electromagnetic case when only electric dipoles are induced. Consider a velocity potential,

$$
\phi=\partial V / \partial x
$$

for the incident and reflected wave in the case of a smooth surface where $V$ is given by (4.5) and where $c$ now characterizes the velocity of sound. Near the reflecting surface $(z=0)$ the tangential velocity component is in the $x$ direction and its value is

$$
u=\partial \phi / \partial x=\partial^{2} V / \partial x^{2} .
$$

Consider, now, a hemispherical boss in a total velocity field $U$ parallel with the $x$ direction. The boundary condition to be satisfied that the normal velocity be zero at the surface of the hemisphere requires that a dipole source be placed at the center of the boss, with its axis along the $x$ direction. The field radiated by this dipole is represented by the velocity potential

$$
\phi_{1}=-M \frac{\partial}{\partial x}\left(\frac{e^{-i k r}}{r}\right)
$$

where

$$
M=\frac{1}{2} U a^{3},
$$

and $r$ is the distance to the dipole source. Therefore, $N$ such dipoles per unit area over the plane $z=0$ radiate a field represented by the velocity potential

$$
\phi^{\prime}=-N \iint M \frac{\partial}{\partial x}\left(\frac{e^{-i k r}}{r}\right) d S .
$$

This is also represented by the scalar

$$
V^{\prime}=-N \iint \frac{e^{-i k r}}{r} d S
$$

with $\phi^{\prime}=\partial V^{\prime} / \partial x$. As pointed out in Sec. 3 we have the property

$$
\left(\partial V^{\prime} / \partial z\right)=-2 \pi N M
$$

at $z=0$. Now the velocity $U$ is the sum of the components due to $V$ and $V^{\prime}$, namely

$$
U=\left(\partial^{2} / \partial x^{2}\right)\left(V+V^{\prime}\right)
$$

Substituting in the expression for $M$ and in (6.13) we find the boundary condition

with

$$
\left(\partial V^{\prime} / \partial z\right)=-\sigma\left(\partial^{2} / \partial x^{2}\right)\left(V+V^{\prime}\right),
$$

$$
\sigma=\pi N a^{3} .
$$

This is identical with (6.3). Hence, the case of the acoustic wave is the same as the electromagnetic 
problem treated in this section. It leads to expression (6.7) for the phase of the reflected wave. The same drastic phase reversal occurs at grazing incidence, and this feature is therefore not restricted to electromagnetic phenomena.

\section{INFLUENCE OF NONRADIATIVE CLOSE RANGE INTERACTION}

In the preceeding derivation we have assumed that the bosses are sufficiently far apart so that the nonradiative interaction, electrostatic and magnetostatic, may be neglected. We shall now briefly investigate the magnitude of this interaction and show that it may easily be introduced into the present treatment without modifying substantially the theory. We continue to assume that the wavelength is large relative to the size of the bosses and their distance from each other. If this is not the case the results may still be considered as approximate within certain limits. The principle applied here is derived from a well-known property, which is discussed at length in reference 1 , that in a region sufficiently small, relative to the wavelength, the field may be evaluated entirely by magnetostatic and electrostatic theory. The singularities, dipoles and multipoles induced in the roughness by the local field are completely determined by the local geometry. We shall calculate only the dipole singularities which constitute the major contribution for hemispherical bosses which are not extremely close together. In principle there is no difficulty in including in the present theory the effect of higher order multipoles associated with other shapes than the hemisphere, or with very close proximity. Consider then an electric field $E$ normal to the plane of reflection. This field considered as a uniform electrostatic field induces vertical electric moments of the same direction and of magnitude $M$ in the bosses. Let us assume that the center of the bosses are located at an average distance $b$ from each other. Then a particular boss located at a point $P$ is surrounded on the average by six electric moments located on a circle of radius $b$. The vertical electric field induced at $P$ by the six surrounding moments is directed in opposite direction to $M$ and has the magnitude $6 M / b^{3}$. The next row of induced moments is on the circle of radius $2 b$. Further rows are on the circles of radius $n b$ and contain $n$ moments. The total induced field at $P$, plus the imposed field $E$ is therefore, ${ }^{8}$

$$
E^{\prime}=E-\frac{6 M}{b^{3}} \sum_{1}^{\infty}\left(\frac{1}{n^{2}}\right)=E-\pi^{2} \frac{M}{b^{3}} .
$$

Substituting the value

$$
M=E^{\prime} a^{3}
$$

${ }^{8}$ For the values of the series $\Sigma\left(1 / n^{2}\right)$ see Jahnke and Ende Table of Functions (B. G. Teubner, Berlin, 1933), p. 322. for the induced dipole we find,

with

$$
E^{\prime}=\frac{1}{\kappa_{1}} E
$$

$$
\kappa_{1}=1+\left(\pi^{2} a^{3} / b^{3}\right) .
$$

A similar argument applies to the magnetic field. The value of this field $H^{\prime}$ induced at the center of a boss by the imposed field and the interaction of nearby dipoles is

$$
H^{\prime}=\frac{1}{\kappa 2} H,
$$

with

$$
\kappa_{2}=1+\frac{\pi^{2} a^{3}}{4 b^{3}} .
$$

The derivation and value of $\kappa_{2}$ is identical with the value obtained for the acoustic case and is discussed extensively in reference 3 .

The reflection theory may then be developed exactly along previous lines except that Eqs. (3.3) are replaced by,

$$
\begin{gathered}
\mathbf{M}=\left(\mathbf{E}+\mathbf{E}^{\prime}\right)^{\frac{a^{3}}{\kappa_{1}},} \\
\mathbf{M}^{*}=-\frac{1}{2}\left(\mathbf{H}+\mathbf{H}^{\prime}\right) \underset{\kappa_{2}}{a^{3}} .
\end{gathered}
$$

The boundary condition (4.9) is replaced by

$$
\frac{\partial V^{\prime}}{\partial z}=-\frac{\sigma}{\kappa_{1}}\left(\frac{\partial}{\partial x^{2}}+\frac{1}{2} \frac{\kappa_{1}}{\kappa_{2}} k^{2}\right)\left(V+V^{\prime}\right) .
$$

If we take into account the relations

and

$$
\partial V / \partial z=0 \quad \text { at } \quad z=0
$$

$$
\left(\frac{\partial^{2}}{\partial x^{2}}+\frac{\partial^{2}}{\partial z^{2}}+k^{2}\right)\left(V+V^{\prime}\right)=0 .
$$

we may write the boundary condition (7.6) as

$$
\begin{aligned}
\frac{\partial V_{t}}{\partial z} & =\frac{\sigma}{\kappa_{1}}\left[\frac{\partial^{2}}{\partial z^{2}}+k^{2}\left(1-\frac{1}{2} \frac{\kappa_{1}}{2 \kappa_{2}}\right)\right] V_{t}, \\
V_{t} & =V+V^{\prime} .
\end{aligned}
$$

This is a relation between the first and second normal derivatives on the reflecting surface. Relations (4.12) and (4.15) are replaced respectively by,

$$
C+D=D-\frac{2 i m+\frac{\sigma}{\kappa_{1}}\left[m^{2}-l^{2}+k^{2}\left(\frac{\kappa_{1}}{\kappa_{2}}-1\right)\right]}{2 i m-\frac{\sigma}{\kappa_{1}}\left[m^{2}-l^{2}+k^{2}\left(\frac{\kappa_{1}}{\kappa_{2}}-1\right)\right]},
$$


and

$$
\tan \varphi=-\frac{1}{2} \sigma \frac{k}{\kappa_{1}} \frac{\cos 2 \alpha+\frac{\kappa_{1}}{\kappa_{2}}-1}{\cos \alpha} .
$$

The numerical example considered in Sec. 4 is a rather extreme case where $a / b=1 / 3$. In this case $\kappa_{1}=1.36$, $\kappa_{2}=1.09$. The angle at which the effect of the roughness disappears is not 45 degrees but $\alpha=52$ degrees. The curves in Fig. 6 are slightly modified, but the difference becomes smaller as $a / \lambda$ tends to zero. The angle at which the influence of the roughness vanishes is now slightly dependent on $\kappa_{1} / \kappa_{2}$, i.e., on the close range geometry of the roughness. It tends rapidly to 45 degrees as $a^{3} / b^{3} \rightarrow 0$. The dissappearance of the effect of the roughness depends on the mutual cancellation of the radiation because of the electric and magnetic dipoles. The magnitude of these induced dipoles depends on $\kappa_{1}$ and $\kappa_{2}$, and the formula indicates that the effect is a function of the ratio $\kappa_{1} / \kappa_{2}$.

In general, the calculation of induced dipoles derived from the nonradiative local interaction, i.e., by solving Laplace's equation, yields a method of evaluating the radiation resulting from any type of small roughness, and is not restricted to hemispherical bosses. Induced multipoles may also be considered if we introduce a boundary condition of the wave equation which is more general than relation (7.8) and involves derivatives higher than the second.

Close range correlation between size and distance of the bosses may be introduced in the calculation of $\kappa_{1}$ and $\kappa_{2}$ if the distribution of the roughness is statistical. Finally, asymmetry may be considered both in the statistical distribution and the shape of the roughness by introducing tensor relationships between the local field and the induced moments. The latter case should be of practical importance in determining the directional effect of ocean waves on radio transmission at grazing incidence.

\section{RESULTS AND CONCLUSIONS}

(1) A simple theory has been developed for the reflection of electromagnetic waves on a rough surface. The roughness is represented by hemispherical bosses on a plane, the size and mutual distance of the bosses being small relative to the wavelength. The case of an acoustic plane wave is also discussed and shown to be analogous to the reflection on an imperfect conductor.

(2) The theory is also extended to show that the nonradiative interaction between bosses may be taken into account by the introduction of parameters which depend on the short range geometry.

(3) The effect of the roughness is found to be equivalent to a new type of boundary condition for the wave equation. This opens the possibility of treatment of reflection problems for nonplanar waves, such as those emanating from sources at finite distance, and diffraction around bodies with a rough surface. In particular it yields the solution for the reflection on a rough surface for waves emanating from a Hertzian dipole. This solution was developed in reference 2 and will be published at a later date. The case of an acoustic point source was treated in reference 3.

(4) The reflected wave has a phase which depends on the roughness and the angle of incidence. Simple formulas are given for this phase angle for the cases of vertical and horizontal polarization.

(5) For the case of vertical polarization and grazing incidence, the roughness, even if it is small, causes a drastic phase reversal of the reflected wave. The grazing angles within which this effect is appreciable can be evaluated from Fig. 6 . This effect does not occur for horizontal polarization.

(6) At normal incidence, contrary to expectation, the effect of the roughness is to lower the effective surface of reflection below the original smooth plane. This is a consequence of the fact that only induced dipoles are taken into account, and that the induced electric dipole disappears for this case while the magnetic dipole remains. A modification of this effect would occur in the expected direction if we took into account the higher-order, induced-electric multipoles as suggested in Sec. 7 .

(7) At a critical angle of incidence in the vicinity of 45 degrees, for vertical polarization and perfect conductivity, the effect of the roughness disappears. This is due to mutual cancellation of the radiation of the electric and magnetic dipoles induced in the roughness by the incident field. The critical angle is exactly 45 degrees if the bosses are sufficiently far apart, and somewhat higher than 45 degrees if this is not the case, depending on the'short range geometry and distribution of the roughness.

(8) The present treatment is readily applicable not only to a roughness represented by hemispherical bosses but to much more general types. Bosses which are not circular in shape will introduce different dipoles in each principal direction leading to anisotropy of the reflection. Short range interaction and its dependence on the correlation function for the distance between protuberance may be introduced. This may include anisotropic reflection due to anisotropy in the statistical distribution. Radio transmission as influenced by gravity waves at the ocean surface is open to simple treatment by such methods. Higher order multipoles induced in nonspherical shapes, or by close proximity may also be introduced in the theory. This would lead to a boundary condition involving derivatives higher than the second in finite or infinite number, or to transcendental functions of the derivative operators.

(9) The coherent scatter is the object of the present treatment. Its separation from the incoherent scatter achieves a clarification of the more general mathematical treatment, and suggests the possibility of evaluating the latter as a perturbation of the present simple results. 